\title{
In vitro-evolved peptides bind monomeric actin and mimic actin-binding protein thymosin- $\beta 4$
}

\author{
Raphael J. Gübeli, ${ }^{1,5}$ Davide Bertoldo, ${ }^{1,5}$ Kenji Shimada, ${ }^{2}$ Christian B. Gerhold, ${ }^{2}$ Verena Hurst, ${ }^{2,3}$ Yuichiro \\ Takahashi, ${ }^{4}$ Kai Harada, ${ }^{4}$ Ganesh K. Mothukuri, ${ }^{1}$ Jonas Wilbs, ${ }^{1}$ Masahiko Harata, ${ }^{4}$ Susan M. Gasser ${ }^{2,3}$ and \\ Christian Heinis ${ }^{1, *}$ \\ ${ }^{1}$ Institute of Chemical Sciences and Engineering, School of Basic Sciences, Ecole Polytechnique Fédérale \\ de Lausanne (EPFL), CH-1015 Lausanne, Switzerland. \\ ${ }^{2}$ Friedrich Miescher Institute for Biomedical Research, Maulbeerstrasse 66, 4058 Basel, Switzerland. \\ ${ }^{3}$ University of Basel, Faculty of Natural Sciences, 4056 Basel, Switzerland. \\ ${ }^{4}$ Laboratory of Molecular Biology, Tohoku University, Tsutsumidori-Amamiyamachi 1-1, Aoba-ku, Sendai \\ 981-8555, Japan. \\ ${ }^{5}$ Authors contributed equally
}

*Correspondence should be addressed to C.H. E-mail: christian.heinis@epfl.ch

\section{KEYWORDS}

Actin, bicyclic peptide, F-actin, G-actin, peptide, phage display, thymosin 


\section{SUMMARY}

Actin is the most abundant protein in eukaryotic cells and is key to many cellular functions. The filamentous form of actin (F-actin) can be studied with help of natural products that specifically recognize it, as for example fluorophore-labeled probes of the bicyclic peptide phalloidin, but no synthetic probes exist for the monomeric form of actin (G-actin). Herein, we have panned a phage display library comprising more than 10 billion bicyclic peptides against $\mathrm{G}$-actin and isolated binders with low nanomolar affinity and greater than 1000 -fold selectivity over F-actin. Sequence analysis revealed a strong similarity to a region of thymosin$\beta 4$, a protein that weakly binds $\mathrm{G}$-actin, and competition binding experiments confirmed a common binding region at the cleft between actin subdomains 1 and 3. Together with F-actin-specific peptides that we isolated too, we evaluated the G-actin peptides as probes in pull-down, imaging and competition binding experiments. While the F-actin peptides were applied successfully for capturing actin in cell lysates and for imaging, the G-actin peptides did not bind in the cellular context, most likely due to competition with thymosin- $\beta 4$ or related endogenous proteins for the same binding site. 


\section{INTRODUCTION}

The dynamic interchange between the monomeric and polymeric forms of actin is key for many cellular functions, including cell locomotion, division and growth. Performing these functions demands strict regulation of the spatial and temporal organization of these two forms - the monomeric G-actin (globular) or the linear polymer microfilament called F-actin (filamentous). ${ }^{1,2}$ This is facilitated on many levels by a myriad of accessory proteins that regulate filament dynamics, such as rate of actin polymerization and depolymerization, the balance between the concentration of monomeric and filamentous actin within the cell, interfilament interactions, and filament branching. ${ }^{3}$

The study of the actin cytoskeleton and its function in various cellular processes has benefited from natural product toxins that bind F-actin and interfere with different steps in the polymerization or depolymerization. ${ }^{4}$ The F-actin binding toxins phalloidin and jasplakinolide are used to stabilize filamentous actin structures, and fluorescein conjugates of these two toxins are used to image F-actin by microscopy. ${ }^{5}$ Phalloidin is a non-cell permeable bicyclic heptapeptide derived from the poisonous mushroom Amanita phalloides that is widely used to visualize the actin cytoskeleton in fixed cells. Jasplakinolide is a cell permeable macrocycle used as a conjugate with the fluorophore silicon rhodamine for imaging the actin cytoskeleton in living cells. ${ }^{6}$ Based on their broad application as research tools, F-actin-targeting molecules and fluorophore conjugates thereof are offered by several commercial providers as research reagents.

However, for G-actin, the monomeric form of actin, synthetic small molecule or peptidic ligands that can easily be labeled with fluorophores by suitable chemical handles do not exist. A range of natural macrocyclebased toxins bind G-actin, such as mycalolide B, swinholide, or halichondramide. ${ }^{7-9}$ Unfortunately, these compounds can only be obtained by extraction from natural sources or through complex chemical synthesis procedures, which complicates the generation of fluorescent derivatives. Proteins that bind G-actin exist and are used as affinity reagents for the monomeric actin, but only after permeabilization of the cell membranes. ${ }^{10}$ The protein DNase I binds G-actin with high affinity ${ }^{11,12}$ and has been applied to stain G-actin. DNase I is still not an ideal reagent for G-actin imaging, though, as newer studies show that it also binds to F-actin. ${ }^{13,14}$ Vitamin D-binding protein (DBP) binds with high affinity and selectivity to G-actin ${ }^{15}$ and is currently the preferred choice for the imaging of G-actin in fixed cells. ${ }^{14}$ One limitation of DBP is that it binds a large surface of actin around the subdomain $3^{16,17}$ and thus competes with functionally important G-actin binding proteins, such as profilin and gelsolin.

The fact that F-actin-specific bicyclic peptides were successfully evolved in nature was motivation for us, and we herein sought to develop synthetic G-actin-specific ligands based on bicyclic peptides too, by employing artificial evolution in a test tube. Towards this end, we screened large combinatorial libraries of 
bicyclic peptides using an approach based on phage display. ${ }^{18}$ Bicyclic peptides are based on two macrocyclic rings that can engage with target proteins, and can generate molecules binding with a high affinity and selectivity to protein targets of choice. ${ }^{19}$ Through this method, we developed peptide probes that bound with nanomolar affinities to G-actin and were over 1000-fold more selective for the monomeric Gactin than for F-actin. In this work, we also identified new bicyclic peptides that are selective for F-actin. Overall, these probes can be conjugated to fluorophores for imaging applications, competitive binding studies, or for immobilization by chemical linkers for purification or pull-down experiments. Such molecules could potentially offer a powerful tool to quantify, track, and visualize the pool of monomeric actin in cells and to shed additional light on its role in various biological functions. ${ }^{10}$ 


\section{RESULTS AND DISCUSSION}

\section{Selection of bicyclic peptides binding to G-actin}

Bicyclic peptide phage display libraries are generated by displaying random linear peptides containing three cysteines on the tip of filamentous phage. These peptides are cyclized by reacting the cysteine side chains with chemical linkers, such as those shown in Figure $1 \mathrm{~A} .{ }^{18}$ To generate bicyclic peptides binding to G-actin, we used a library containing more than $4 \times 10^{9}$ different peptides of the format $A C X_{6} C X_{6} C G$ ( $X$ indicates random amino acids, $A=$ alanine, $G=$ glycine; top line in Figure $1 B)^{18}$ and cyclized them in separate reactions with 1,3,5-tris(bromomethyl)benzene (TBMB), 1,3,5-triacryloyl-1,3,5-triazinane (TATA), and $N, N^{\prime}, N^{\prime \prime}$-(benzene-1,3,5-triyl)-tris(2-bromoacetamide) (TBAB ${ }^{20}$ to obtain three libraries with a total diversity exceeding 12 billion bicyclic peptides.

For the affinity selections, we immobilized biotinylated rabbit skeletal muscle actin on streptavidin or neutravidin magnetic beads. During the immobilization, actin was kept at a low concentration in G-buffer to favor its immobilization in the monomeric form (see materials and methods section for the conditions). Excess biotinylated actin was removed to prevent polymerization with immobilized units. The binding of biotinylated actin to streptavidin is essentially irreversible, and we thus hoped that actin could not polymerize after immobilization, although actin monomers that were bound to the same streptavidin/neutravidin tetramer could potentially interact with each other to form dimeric or multimeric structures. The three libraries were subjected to three consecutive rounds of phage selection and propagation. Streptavidin (round 1 and 3 ) and neutravidin beads (round 2) were used alternatingly to prevent enriching for binders to these proteins. ${ }^{21}$ The number of phage particles captured in the $3^{\text {rd }}$ round in selections with all three libraries was increased 100-fold over the negative control (no actin immobilized), suggesting that actin-specific bicyclic peptides were enriched (Table S1).

We sequenced the peptide-coding DNA of several hundred thousand isolated phage particles by nextgeneration sequencing, ranked the peptides containing three cysteines according to their abundance, and compared the sequences using a previously developed software to identify peptides that share similar sequences ${ }^{22}$. Comparison of the 200 most abundant peptides revealed seven consensus sequences, which are sequences that are shared by several different but similar peptides of a group (Figure 1B). Additional peptides belonging to the seven consensus groups were identified by searching all identified peptides for the presence of key amino acids characteristic of the consensus groups. Peptides sharing a similar sequence are likely forming the same key molecular interactions with the target by binding to the same surface region. Conversely, peptides of different consensus groups form different molecular contacts. Having identified as many as seven consensus sequences, it was likely that ligands to several different surface regions of actin were isolated. 


\section{Binding properties of bicyclic peptides}

To measure the binding affinity for G- and F-actin by fluorescence polarization, we synthesized representative peptides of the different consensus groups and linked fluorescein to the $\mathrm{N}$-termini of all peptides. The bicyclic peptides at a concentration of $10 \mathrm{nM}$ were incubated in either G- or F-buffer with actin at concentrations ranging from $15 \mathrm{nM}$ to $2 \mu \mathrm{M}$. Among the 19 peptides that we tested, 13 showed binding to either G- and/or F-actin (Figure 1). Bicyclic peptides of the second consensus group bound with high affinity and selectivity to G-actin, displaying dissociation constants ( $\left.K_{d} S\right)$ as low as $22 \mathrm{nM}$ (peptide A11) and a greater than 1000-fold selectivity over F-actin. A bicyclic peptide tested from the sixth group, A18, bound with moderate affinity to $\mathrm{F}$-actin $(1.97 \mu \mathrm{M})$ and showed no binding to $\mathrm{G}$-actin. Bicyclic peptides from several groups bound both forms of actin. The F-actin-specific binders were potentially enriched due to traces of Factin that were formed despite our efforts of immobilizing actin on magnetic beads in its monomeric form. It is tempting to speculate that peptides with higher affinity and selectivity for F-actin may be developed in future if combinatorial libraries of cyclic peptide were panned against the polymeric form of actin immobilized on beads.

Of highest interest for us were the G-actin-specific peptides of the second consensus group, such as peptide A10, for example, that bound G-actin with a $K_{d}$ of $31 \mathrm{nM}$ (Figure $2 \mathrm{~A}$ ). The strong binding of $\mathrm{A} 10$ to actin in G-buffer could be reversed by converting G-actin into F-actin through the addition of F-buffer and phalloidin, the former triggering actin polymerization and the latter stabilizing the polymeric F-actin (Figure 2B). Two of the peptides of the second consensus group, $A 9$ and $A 11$, had the same amino acid sequence but were cyclized with different linkers - A9 with TBMB and A11 with TATA. The peptide cyclized with TBMB bound G-actin with much weaker affinity $\left(K_{d}=700 \mathrm{nM}\right)$ than the TATA-cyclized peptide $\mathrm{A} 11\left(K_{d}=22 \mathrm{nM}\right.$; Figure S1), which showed the importance of the cyclization linker for binding - validating the strategy of screening the three different sub-libraries in parallel.

\section{Sequence homology between peptides and thymosin- $\beta 4$}

We next compared the seven consensus sequences with the amino acid sequences of actin-binding proteins. This revealed a strong sequence similarity between the second consensus group (peptides A9 to A12 sharing the sequence KSRLGCF) and a sequence of four amino acids in the actin-sequestering protein thymosin- $\beta 4$ (T $\beta 4 ; \mathrm{KSKL}$; Figure $3 \mathrm{~A})$. T $\beta 4$ is an intrinsically disordered protein of 43 amino acids that binds monomeric actin in cells and regulates polymerization. ${ }^{23}$ It addition to its intracellular role, T $\beta 4$ showed diverse activities in tissue protection and repair, which has led to its therapeutic evaluation in animals and humans. Cellular concentrations of $\mathrm{T} \beta 4$ are in the $100-$ to $500-\mu \mathrm{M}$ range, and the binding affinity $\left(K_{\mathrm{d}}\right)$ for ATP- and ADP-bound actin are in the ranges of $0.2-9 \mu \mathrm{M}$ and $80-100 \mu \mathrm{M}$, respectively. ${ }^{24-28}$ The four-amino 
acids KSKL of T $\beta 4$ fall into an actin interaction motif termed WH2 (Wiscott-Aldridge syndrome protein homology domain 2) repeat that is shared by many G-actin sequestering and filament assembly proteins. ${ }^{29,30}$ Mutational analysis of this region in T $\beta 4$ confirmed its importance for actin monomer binding. ${ }^{27,29}$ Crystal structures of $T \beta 4$ and hybrids of $T \beta 4$ bound to actin 27,28 show that the four amino acids KSKL form a turn and that the side chains of the first (Lys14) and last (Leu17) of the four amino acids form contacts with the actin surface (Figure 3B). We performed an alanine scan with one of the peptides, A11, to test the importance of these two amino acids. Mutating the Lys in A11 reduced the binding affinity 20 -fold and mutating the Leu led to complete loss of binding, showing that these two amino acids form key interactions (Figure 3C). The alanine scan identified several other amino acids in A11 that are critical for binding but that are not present in T $\beta 4$. Based on the sequence similarity between the consensus region of the bicyclic peptides and $\mathrm{T} \beta 4$ and the finding that these amino acids form key interactions, we concluded that the first ring of the bicyclic peptide most likely mimics the binding mode of $T \beta 4$ in this region. The peptide A10, that was isolated during the phage panning with TBMB linker and is sharing a high sequence similarity with $A 11$, does not contain the Lys of the consensus motif but Gly-Pro instead, raising the question if the Lys is as important as suggested by the alanine scan. Replacement of the Lys in A11 with Gly-Pro or Pro reduced the peptide's affinity for G-actin 170- and 12-fold, respectively, confirming the importance of Lys for A11 (Figure S2). We concluded that the Gly-Pro sequence found in A10 had most likely evolved because it was selected with a different linker (TBMB) than A11 (TATA linker). A linear version of A11 did not bind to Gactin (Figure $3 C$ ), further showing that the bicyclic configuration is important. Overall, we had thus not only mimicked nature by evolving bicyclic peptide for binding to actin, but even found peptides that have a similar binding motif as a natural actin-binding protein. Additionally, these actin-binding ligands are the first ones "artificially" developed in a test tube, to our knowledge.

\section{Binding competition with $\mathrm{T} \beta 4$ and mycalolide $B$}

We next tested if the G-actin-specific bicyclic peptides compete with T $\beta 4$ for binding to G-actin, as was expected based on the sequence similarity. We incubated the fluorescein-labeled peptide A10 at a concentration of $10 \mathrm{nM}$ with increasing G-actin concentrations, either in the presence or absence of $20 \mu \mathrm{M}$ $\mathrm{T} \beta 4$, and found that the actin-sequestering protein hindered actin binding to A10 (Figure 4). We next performed the same fluorescence competition experiment with two other actin-binding proteins, DNase I and profilin, and two actin-targeting toxins, mycalolide B and latrunculin B - all molecules for which the binding sites on actin are known, as shown in Figure 4. DNase I did not compete with the binding of A10 to G-actin, which was expected based on its binding site that is a flexible loop in actin subdomain 2. ${ }^{12}$ Profilin did not block the binding of $\mathrm{A} 10$, but appears to reduce its affinity. Profilin binds to the hydrophobic cleft between the actin subdomains 1 and 3 that is close to the expected binding region of $A 10$ and potentially affects bicyclic-peptide binding by imposing conformational changes in actin. Latrunculin $\mathrm{B}$, which binds to the ATP-binding cleft of actin, did not inhibit the binding of the bicyclic peptide. Finally, the trisoxazole 
macrocycle mycalolide $B$ fully blocked the binding of $A 10$ to actin. Mycalolide $B$ is known to bind to a large hydrophobic patch on actin that leads into the narrow cleft separating subdomains 1 and 3 at the barbed end. This is essentially the same region where the 4-amino acid stretch KSKL of T $\beta 4$ binds. Taken together, the competition experiments confirmed the hypothesized binding site of the $\mathrm{G}$-actin-specific bicyclic peptides at the hydrophobic patch of actin that leads into the narrow cleft separating subdomains 1 and 3 at the barbed end. The finding that $\mathrm{A} 10$ can bind to profilin-bound G-actin was interesting, as it suggested that the bicyclic peptide can be used as an affinity reagent to distinguish between G-actin/T $\beta 4$ and G-actin/profilin complexes, and thus between the two largest G-actin pools in cells. ${ }^{10}$

\section{Application of peptides in pull-down experiments}

Peptides are suited as affinity reagents in pull-down experiments, as they can easily be synthesized with chemical linkers or tags and can be immobilized on solid supports, such as magnetic beads. We hypothesized that the G-actin-specific peptides could be used to capture and quantify G-actin that is not bound by $T \beta 4$, and that F-actin-specific peptides could be used to pull-down any form of F-actin. We immobilized the fluorescein-labeled peptides on beads coated with an anti-fluorescein antibody, incubated the beads with fresh budding yeast (W303) or mammalian cell lysates (HeLa), and analyzed if actin was captured by SDS-PAGE (Figure 5). We performed this experiment with the G-actin-specific peptides, the Factin-specific peptides, and the peptides that bind both forms of actin. With the G-actin-specific peptides (A4, A9, A10, A12), no actin was enriched (Figure 5A and 5B). In case of the peptide A4, it is likely that the rather weak binding affinity for $\mathrm{G}$-actin $\left(K_{d}\right.$ of $\left.4.6 \mu \mathrm{M}\right)$ was not sufficient to capture the monomeric protein. In the case of peptides A9, A10 and A12, we speculated that occupation of the peptide-binding site of Gactin by sequestering proteins prevented capture of actin. A control experiment performed with purified Gactin showed that the protein is captured by G-actin-specific peptide (A10) using the described pull-down protocol (Figure $5 \mathrm{C}$ ), and indicated that the problem with the G-actin pull-downs were indeed most likely resulting from competing components in cell lysates. With the F-actin-specific peptides (A3, A18) and peptides with mixed specificity (A15, A16), actin was efficiently captured (Figure 5A and 5B).

\section{F-actin imaging using bicyclic peptides}

Given that the G-actin-specific bicyclic peptides did not capture actin from cell lysates, we expected that microscopic imaging would be impossible with the G-actin-specific probes. Indeed, fluorescence imaging of fluorescein-labeled G-actin-specific bicyclic peptide did not show any particular staining pattern, and we thus focused on the evaluation of the phage-selected F-actin-specific bicyclic peptide A18. Fixed HeLa cells stained with the fluorescein-labeled A18 showed the characteristic actin structures (Figure 6, left panel, green). Co-staining with iFluor 647-phalloidin gave the same structures (Figure 6, middle panel, red), and the fluorescence signals overlapped perfectly (Figure 6, right panel). In a control with latrunculin B that 
dissociates actin fibers, the actin filaments were no longer seen, further confirming that the bicyclic peptides specifically localized to F-actin. The specific staining of F-actin with A18 was not necessarily expected, considering its rather weak binding affinity $\left(K_{d}=2 \mu \mathrm{M}\right)$. The experiment with the F-actin binders showed that phage-selected actin-specific bicyclic peptides are suited for imagining applications.

\section{Measuring the binding of natural actin ligands}

The high affinity and robust performance in fluorescence polarization assays suggested that the bicyclic peptides may be used as probes to measure the affinity of natural ligands that bind to the same region. For these experiments, a fluorescence-labeled bicyclic peptide at a low concentration was incubated with Gactin at a concentration slightly above the $K_{d}$ of the actin-peptide interaction to bind and polarize 50 to $70 \%$ of the probe. The natural ligand was then added to the complex, the concentration determined at which $50 \%$ of the fluorescent peptide is displaced, and the dissociation constant was calculated. Several natural proteins and natural product toxins bind to the actin cleft between subdomains 1 and 3 . We chose to quantify the binding affinity of the actin-binding protein $T \beta 4$ and the marine sponge toxins swinholide, mycalolide $B$ and halichondramide.

For the fluorescence polarization competition experiment, we used the fluorescein-labeled bicyclic peptide A20, which is an Asn11Ala mutant of the bicyclic peptide A11 that was identified in the alanine scan described above and that has a slightly higher affinity $\left(K_{d}=5 \pm 2 \mathrm{nM}\right)$. A probe with this affinity promised the measurement of dissociation constants in the micromolar to low nanomolar range. The peptide A20 also displayed good binding affinities in presence of the physiologically relevant cation $\mathrm{Mg}^{2+}$ and with bound ATP $\left(K_{d}=14 \pm 4 \mathrm{nM}\right)$ or ADP ( $K_{d}=21 \pm 0.4 \mathrm{nM}$; Figure S3 and Table S2). In competition binding experiments, we determined the concentrations at which $\mathrm{T} \beta 4$ and the three marine toxins displace $50 \%$ of A20 (Figure 7) and calculated the dissociation constants. In presence of magnesium, T $\beta 4$ bound ATP-G-actin with a $K_{d}$ of $127 \pm 11 \mathrm{nM}$ and ADP-G-actin with > 20-fold weaker affinity. The three marine toxins all bound better to ADP-G-actin than to ATP-G-actin and with dissociation constants below $100 \mathrm{nM}$ (Table 1). For mycalolide $B$ and halichondramide, the dissociation constant could be estimated but not determined accurately because they bound with a higher affinity than the bicyclic peptide probe $\left(K_{\mathrm{d}}<10 \mathrm{nM}\right)$. 


\section{CONCLUSIONS}

We report the successful development of peptide-based ligands of actin using structurally diverse phagedisplay libraries that comprise billions of different bicyclic peptides. We identified a group of peptides that displayed low nanomolar affinity for G-actin and that did not bind F-actin. All of the peptides have a good solubility and can he handled easily, both properties that explain their robust performance in binding assays. These are also the first G-actin-specific binding ligands that are simple to modify, which make them a unique tool for studying the pools of non-sequestered monomeric actin. While we aimed primarily at developing binders of G-actin, some of the isolated peptides bind both forms and some preferentially bind F-actin. In the end, this discovery provided us with a set of actin-binding ligands, all equally as facile to modify and behaving in a similar fashion, that can be used for a variety of actin detection or binding assays. It is possible that different ligands will be required for $\mathrm{G}$-actin measurements in specific scenarios since there are so many different binding sites on G-actin. To make G-actin ligands binding to a specific location on the protein, it would be possible to modify the immobilization and panning conditions to change how G-actin is detected. For instance, specific elutions that use known G-actin proteins to outcompete for a binding site could provide peptides that bind to other known sites on the protein. Alternatively, it might also be possible to improve the binding affinity of the herein developed G-actin binders so that they could compete with the highly abundant but less tightly binding actin sequestering proteins. Overall, our herein presented method for developing ligands to the various forms of actin, as well as the high-affinity and form-specific peptides that were developed through this method, provide an easy way for detecting purified G-actin in vitro, for example in competition binding assays, or for detecting and measuring the pools of F-actin in cells or cell lysates. 


\section{MATERIALS AND METHODS}

\section{Immobilization of G-actin on magnetic beads}

Lyophilized biotinylated rabbit skeletal actin (AB07, Cytoskeleton) was dissolved in milliQ water to obtain a concentration of $10 \mathrm{mg} / \mathrm{ml}$ in a buffer containing $5 \mathrm{mM} \mathrm{Tris-HCl} \mathrm{pH} \mathrm{8.0,0.2} \mathrm{mM} \mathrm{CaCl}, 0.2 \mathrm{mM} \mathrm{ATP}, 5 \%$ sucrose, and $1 \%$ dextran. Around $5 \mu \mathrm{g}$ of the protein were diluted in $0.5 \mathrm{ml}$ of G-buffer $(5 \mathrm{mM} \mathrm{Tris-HCl} \mathrm{pH}$ 7.8, $0.1 \mathrm{mM} \mathrm{CaCl}_{2}, 0.2 \mathrm{mM} \mathrm{ATP,} 1 \mathrm{mM} \mathrm{DTT}$ ) and incubated for $10 \mathrm{~min}$ with $50 \mu$ streptavidin or neutravidin magnetic beads on a rotating wheel $(10 \mathrm{rpm})$. The beads were then collected with a magnet on the wall of a tube, and the excess actin was removed by pipetting out the supernatant. The beads were washed twice using the same procedure and were resuspended in the original volume of buffer.

\section{Phage display selection}

Phage of the $6 \times 6$ peptide library (sequence: $A C X_{6} C X_{6} C G$, where $X$ indicates random amino acids, $A=$ alanine, $G=$ glycine $)^{18}$ were produced and purified on a $1.5 \mathrm{~L}$ scale. Peptides displayed on the phage were reduced with tris(2-carboxyethyl)phosphine (TCEP), and the reducing agent was removed by filtering the phage as previously described. ${ }^{18}$ The phage were split into three tubes to cyclize the peptides with 1,3,5Tris(bromomethyl)benzene (TBMB, $20 \mu \mathrm{M}$ ), 1,3,5-triacryloyl-1,3,5-triazinane (TATA, $150 \mu \mathrm{M}$ ), and N,N',N"(benzene-1,3,5-triyl)tris(2-bromoacetamide) (TBAB, $40 \mu \mathrm{M}$ ) as described previously. ${ }^{20}$ Each of the three bicyclic peptide phage libraries was subjected to affinity selections as described previously, ${ }^{18}$ except that the binding buffer was changed to $10 \mathrm{mM}$ HEPES pH 7.9, $100 \mathrm{mM} \mathrm{KCl}, 2 \mathrm{mM} \mathrm{CaCl}$, 2.5\% glycerol, $0.2 \mathrm{mM}$ ATP, and $1 \mathrm{mM}$ DTT. The blocking buffer and washing buffer were also changed accordingly. For each selection, $5 \mu \mathrm{g}$ of biotinylated rabbit skeletal muscle actin immobilized on $50 \mu \mathrm{l}$ of magnetic streptavidin (1st and 3 rd round) or neutravidin beads (2nd round) were used. Neutravidin-coated magnetic beads were used in the second round of selection to prevent enrichment for streptavidin-specific peptides. Eluted phage were incubated with $30 \mathrm{ml}$ of exponentially growing TG1 E. coli cells $\left(\mathrm{OD}_{600}=0.4\right)$ for $90 \mathrm{~min}$. Bacteria were plated on large $2 Y T /$ chloramphenicol plates $(30 \mathrm{mg} / \mathrm{ml})$ and incubated at $37^{\circ} \mathrm{C}$. The cells were harvested the next day with $2 \mathrm{YT}$ media and stored in $15 \%$ glycerol at $-80^{\circ} \mathrm{C}$.

\section{Phage DNA sequencing}

For Sanger sequencing, cells from single colonies were grown in $5 \mathrm{ml}$ of LB media containing chloramphenicol (30 mg/ml). The DNA was then isolated and sequenced (Macrogen). For high-throughput sequencing, the cells from the colonies were pooled and then the DNA was isolated. The DNA was processed as described previously and was sequenced by ion torrent sequencing. The data were analyzed using Matlab and pre-written algorithms as described previously. ${ }^{22}$ 


\section{Fluorescence polarization binding assay}

More than 99\% pure rabbit skeletal muscle actin (AKL99, Cytoskeleton) was used in all fluorescence polarization binding assays. The protein was depolymerized for $1 \mathrm{~h}$ on ice in G-buffer containing $0.01 \%$ Tween-20 and serially diluted in the same buffer (twofold, starting from $4 \mu \mathrm{M}$ ). Then, $20 \mu \mathrm{l}$ of each dilution were transferred to wells of a black Greiner Bio-one 96-well flat-bottom half-area polystyrene microtiter plate, and $20 \mu \mathrm{l}$ of fluorescein-labeled peptide $(20 \mathrm{nM})$ in G-buffer containing $0.01 \%$ Tween-20 were added to each well and incubated for $5 \mathrm{~min}$. Anisotropy of each solution was measured in a multi-well plate reader (Infinite 200 PRO, Tecan) using a $485 \mathrm{~nm}$ excitation filter and a $535 \mathrm{~nm}$ emission filter.

The dissociation constants $\left(K_{d} \mathrm{~S}\right)$ were determined using a non-linear regression analyses of fluorescence anisotropy $(A)$ versus total concentration of actin $[R]$ T using the following equation:

$$
A=A_{f}+\left(A_{b}-A_{f}\right) * \frac{\left(L_{T}^{*}+K_{d}^{L *}+R_{T}\right)-\sqrt[2]{\left(L_{T}^{*}-K_{d}^{L *}-R_{T}\right)^{2}-4 L_{T}^{*} R_{T}}}{2 L_{T}^{*}}
$$

Where,

$A=$ observed anisotropy value

$A_{b}=$ anisotropy of the bound fluorescent ligand

$A_{f}=$ anisotropy of the free fluorescent ligand

$L_{T}^{*}=$ total added concentration of fluorescent ligand

$K_{d}^{L *}=$ equilibrium dissociation constant for $L_{T}$

$R_{T}=$ total added concentration of receptor

GraphPad Prism 5 software (GraphPad software) was used for the analysis.

The same procedure was used to determine the binding affinity of the peptides in $\mathrm{Mg}^{2+}$ ATP G-buffer, wherein buffer containing $\mathrm{Mg}^{2+}$ and ATP (5 mM Tris- $\mathrm{HCl} \mathrm{pH} 7.8,0.2 \mathrm{mM}$ EGTA, $0.1 \mathrm{mM} \mathrm{MgCl}, 0.2 \mathrm{mM}$ ATP, $1 \mathrm{mM} \mathrm{DTT}$ ) and $0.01 \%$ Tween-20 was used to dilute actin and the fluorescent peptide. For measuring the binding of peptides to $\mathrm{Mg}^{2+} \mathrm{ADP}$ actin, actin was prepared according to the protocol of Pollard. ${ }^{1}$ Briefly, rabbit skeletal muscle actin was diluted to $20 \mu \mathrm{M}$ in G-buffer supplemented with $50 \mu \mathrm{M} \mathrm{MgCl}_{2}$ and $400 \mu \mathrm{M}$ glucose. ATP actin was converted to ADP actin by adding 25 units $/ \mathrm{ml}$ of hexokinase (Sigma) and incubating the sample $72 \mathrm{~h}$ at $4^{\circ} \mathrm{C}$. Actin and fluorescent peptide were diluted in $\mathrm{Mg}^{2+} \mathrm{ADP}$ G-buffer $(5 \mathrm{mM}$ Tris-HCl pH 7.8, 0.2 mM EGTA, 0.1 mM MgCl $2,0.2$ mM ADP, 1 mM DTT) containing 0.01\% Tween-20. 


\section{Pull-down assay}

First, $80 \mu \mathrm{g}$ of anti-fluorescein (FITC) mouse antibody (Jackson Immuno Research, clone IF8-IE4) was coupled with $400 \mu \mathrm{l}$ of Protein G-Dynal beads (Dynal) and cross-linked by dimethyl pimelimidate (Pierce). Then, $30 \mu \mathrm{l}$ of anti-FITC antibody coupled Dynal beads (6 $\mu \mathrm{g}$ antibody equivalent) was incubated with 1.5 nmol of FITC-coupled bicyclic peptide in PBS for $1.5 \mathrm{~h}$, then washed twice with PBS and once with cell lysis buffer (50 mM HEPES pH 7.5, $20 \mathrm{mM} \mathrm{NaCl}, 1 \mathrm{mM}$ EDTA, 0.1\% Triton X-100 containing protease inhibitor cocktail EDTA free [Roche]). W303 yeast cells were exponentially cultured in synthetic complete medium $2 \%$ glucose (SC). The following steps were conducted at $4^{\circ} \mathrm{C}$ : Roughly $5 \times 10^{8}$ yeast cells were resuspended in $400 \mu \mathrm{l}$ of lysis buffer and broken by bead beating $(6.5 \mathrm{M} / \mathrm{sec}, 60$ seconds, 4 times; FastPrep-24, MP Biomedicals) with Zirconia beads. The cell extract was prepared by pelleting (12,000 g, $5 \mathrm{~min}$ ). Then, 100 $\mu \mathrm{l}$ of cell extract was used for one pull-down assay. The bicyclic-peptide-coupled Dynal beads prepared above were incubated with yeast cell extract at $4^{\circ} \mathrm{C}$ with rotation for $1.5 \mathrm{~h}$. Dynal beads were recovered by collecting on a magnetic stand and were resuspended in $0.5 \mathrm{ml}$ lysis buffer and rotated at $4^{\circ} \mathrm{C}$ for $10 \mathrm{~min}$ (washing). This washing step was repeated two more times. The protein bound to the Dynal beads was eluted by $60 \mu \mathrm{l}$ of $100 \mathrm{mM}$ glycine $\mathrm{pH} 2.0,0.1 \%$ TritonX-100, and was neutralized by $3.5 \mu \mathrm{l}$ of $1 \mathrm{M}$ Tris- $\mathrm{HCl}$ $\mathrm{pH}$ 9.5, then mixed with $16 \mu \mathrm{l}$ of $4 \times$ NuPAGE sample buffer (Thermo Fisher Scientific) and heated at $95^{\circ} \mathrm{C}$ for $5 \mathrm{~min}$. Protein samples were subjected to $4-12 \%$ Bolt $^{\mathrm{TM}}$ Bis-Tris Gel (Thermo Fisher Scientific) in MES buffer and were silver stained or Western blotted with anti-actin (clone C4, mab1501, Millipore) or antitubulin (clone DM1A, Sigma). HeLa cells were cultured in DMEM medium supplemented with 10\% FCS, at $37^{\circ} \mathrm{C}$ in $5 \% \mathrm{CO}_{2}$. Hela cells from three $10 \mathrm{~cm}$ dishes cultured at $80 \%$ confluence were collected by a cell scraper and were washed by ice cold PBS. Cells were resuspended in $400 \mu \mathrm{l}$ of lysis buffer, and the total cell extract was prepared with $5.0 \mathrm{M} / \mathrm{sec}, 30 \mathrm{sec}$, three times beat beading as above. Bicyclic peptide pulldown and Western blot assay were conducted as above with $100 \mu \mathrm{l}$ of HeLa cell extract per one pull-down.

\section{Microscopy}

HeLa cells grown on cover slips were treated with DMSO $(<0.025 \%)$ or latrunculin $B(600 \mathrm{nM}, 60 \mathrm{~min}$, ab144291) in cell culture medium (DMEM), fixed with 4\% PFA/PBS for $10 \mathrm{~min}$, permeabilized with $0.1 \%$ Triton X-100/PBS (2 min, RT), blocked with 1\% BSA/PBS (90 min, RT), and stained overnight with $1 \mu \mathrm{M}$ of the fluorescein-labeled peptide $\mathrm{A} 18$ or/and phalloidin (iFluor 647-phalloidin, far red) at $4^{\circ} \mathrm{C}$. After 3 washes in PBS, the cells were mounted with prolong gold antifade and imaged at $100 \times$ magnification. Image stacks were deconvolved with Huygens software and were processed further in Fiji. Stacks were converted to maximum intensity projections. Then, to correct for channel bleed-through, a fixed value was subtracted from each pixel. Negative intensity values were set to zero, and the contrast of all images was enhanced automatically (saturated $=0.35$ ). 


\section{ASSOCIATED CONTENT}

Supporting Information

The Supporting Information is available free of charge at https://pubs.acs.org/XYZ

Supplemental materials and methods, tables $\mathrm{S} 1$ to $\mathrm{S} 3$ and figures $\mathrm{S} 1$ to $\mathrm{S} 4$.

\section{ACKNOWLEDGMENTS}

We are grateful to J. Tanaka, University of the Ryukyus, for the kind donation of halichondramide. We thank

K. Butler and C. Horigome for discussions and feedback on the manuscript. The project was supported by the Swiss National Science Foundation (Sinergia grant: 141945) and the Human Frontiers Science Program (Grant: Actin and actin-related proteins - probing their nuclear function).

\section{DECLARATION OF INTERESTS}

The authors have declared that no conflict of interest exists. 


\section{REFERENCES}

(1) Pollard, T. D. Actin and Actin-Binding Proteins. Cold Spring Harb. Perspect. Biol. 2016, 8 (8), a018226. https://doi.org/10.1101/cshperspect.a018226.

(2) Dominguez, R.; Holmes, K. C. Actin Structure and Function. Annu. Rev. Biophys. 2011, 40 (1), 169-186. https://doi.org/10.1146/annurev-biophys-042910-155359.

(3) Carlier, M.-F.; Shekhar, S. Global Treadmilling Coordinates Actin Turnover and Controls the Size of Actin Networks. Nat. Rev. Mol. Cell Biol. 2017, 18 (6), 389-401.

https://doi.org/10.1038/nrm.2016.172.

(4) Allingham, J. S.; Klenchin, V. A.; Rayment, I. Actin-Targeting Natural Products: Structures, Properties and Mechanisms of Action. Cell. Mol. Life Sci. 2006, 63 (18), 2119-2134. https://doi.org/10.1007/s00018-006-6157-9.

(5) Melak, M.; Plessner, M.; Grosse, R. Actin Visualization at a Glance. J. Cell Sci. 2017, 130 (3), 525-530. https://doi.org/10.1242/jcs.189068.

(6) Lukinavičius, G.; Reymond, L.; D’Este, E.; Masharina, A.; Göttfert, F.; Ta, H.; Güther, A.; Fournier, M.; Rizzo, S.; Waldmann, H.; et al. Fluorogenic Probes for Live-Cell Imaging of the Cytoskeleton. Nat. Methods 2014, 11 (7), 731-733. https://doi.org/10.1038/nmeth.2972.

(7) Klenchin, V. A.; Allingham, J. S.; King, R.; Tanaka, J.; Marriott, G.; Rayment, I. Trisoxazole Macrolide Toxins Mimic the Binding of Actin-Capping Proteins to Actin. Nat. Struct. Mol. Biol. 2003, 10 (12), 1058-1063. https://doi.org/10.1038/nsb1006.

(8) Paterson, I.; Yeung, K.-S.; Ward, R. A.; Cumming, J. G.; Smith, J. D. Total Synthesis of Swinholide A and Hemiswinholide A. J. Am. Chem. Soc. 1994, 116 (20), 9391-9392.

https://doi.org/10.1021/ja00099a091.

(9) Panek, J. S.; Liu, P. Total Synthesis of the Actin-Depolymerizing Agent (-)-Mycalolide A: Application of Chiral Silane-Based Bond Construction Methodology. J. Am. Chem. Soc. 2000, 122 (45), 11090-11097. https://doi.org/10.1021/ja002377a.

(10) Skruber, K.; Read, T.-A.; Vitriol, E. A. Reconsidering an Active Role for G-Actin in Cytoskeletal Regulation. J. Cell Sci. 2018, 131 (1), jcs203760. https://doi.org/10.1242/jcs.203760.

(11) Hitchcock, S. E. Actin Deoxyroboncuclease I Interaction. Depolymerization and Nucleotide Exchange. J. Biol. Chem. 1980, 255 (12), 5668-5673.

(12) Kabsch, W.; Mannherz, H. G.; Suck, D.; Pai, E. F.; Holmes, K. C. Atomic Structure of the Actin: DNase I Complex. Nature 1990, 347 (6288), 37-44. https://doi.org/10.1038/347037a0.

(13) Morrison, S. S.; Dawson, J. F. A High-Throughput Assay Shows That DNase-I Binds Actin Monomers and Polymers with Similar Affinity. Anal. Biochem. 2007, 364 (2), 159-164. 
https://doi.org/10.1016/j.ab.2007.02.027.

(14) Lee, C. W.; Vitriol, E. A.; Shim, S.; Wise, A. L.; Velayutham, R. P.; Zheng, J. Q. Dynamic Localization of G-Actin during Membrane Protrusion in Neuronal Motility. Curr. Biol. 2013, 23 (12), 1046-1056. https://doi.org/10.1016/j.cub.2013.04.057.

(15) Van Baelen, H.; Bouillon, R.; De Moor, P. Vitamin D-Binding Protein (Gc-Globulin) Binds Actin. J. Biol. Chem. 1980, 255 (6), 2270-2272.

(16) Otterbein, L. R.; Cosio, C.; Graceffa, P.; Dominguez, R. Crystal Structures of the Vitamin D-Binding Protein and Its Complex with Actin: Structural Basis of the Actin-Scavenger System. Proc. Natl. Acad. Sci. 2002, 99 (12), 8003-8008. https://doi.org/10.1073/pnas.122126299.

(17) Verboven, C.; Rabijns, A.; De Maeyer, M.; Van Baelen, H.; Bouillon, R.; De Ranter, C. A Structural Basis for the Unique Binding Features of the Human Vitamin D-Binding Protein. Nat. Struct. Biol. 2002, 9 (2), 131-136. https://doi.org/10.1038/nsb754.

(18) Heinis, C.; Rutherford, T.; Freund, S.; Winter, G. Phage-Encoded Combinatorial Chemical Libraries Based on Bicyclic Peptides. Nat. Chem. Biol. 2009, 5 (7), 502-507.

https://doi.org/10.1038/nchembio.184.

(19) Deyle, K.; Kong, X.-D.; Heinis, C. Phage Selection of Cyclic Peptides for Application in Research and Drug Development. Acc. Chem. Res. 2017, 50 (8), 1866-1874.

https://doi.org/10.1021/acs.accounts.7b00184.

(20) Chen, S.; Bertoldo, D.; Angelini, A.; Pojer, F.; Heinis, C. Peptide Ligands Stabilized by Small Molecules. Angew. Chemie Int. Ed. 2014, 53 (6), 1602-1606.

https://doi.org/10.1002/anie.201309459.

(21) Rentero Rebollo, I.; Heinis, C. Phage Selection of Bicyclic Peptides. Methods 2013, 60 (1), $46-54$. https://doi.org/10.1016/j.ymeth.2012.12.008.

(22) Rentero Rebollo, I.; Sabisz, M.; Baeriswyl, V.; Heinis, C. Identification of Target-Binding Peptide Motifs by High-Throughput Sequencing of Phage-Selected Peptides. Nucleic Acids Res. 2014, 42 (22), e169-e169. https://doi.org/10.1093/nar/gku940.

(23) Safer, D.; Sosnick, T. R.; Elzinga, M. Thymosin $\beta 4$ Binds Actin in an Extended Conformation and Contacts Both the Barbed and Pointed Ends †. Biochemistry 1997, 36 (19), 5806-5816. https://doi.org/10.1021/bi970185v.

(24) Weber, A.; Nachmias, V. T.; Pennise, C. R.; Pring, M.; Safer, D. Interaction of Thymosin .Beta.4 with Muscle and Platelet Actin: Implications for Actin Sequestration in Resting Platelets. Biochemistry 1992, 31 (27), 6179-6185. https://doi.org/10.1021/bi00142a002.

(25) Carlier, M. F.; Jean, C.; Rieger, K. J.; Lenfant, M.; Pantaloni, D. Modulation of the Interaction 
between G-Actin and Thymosin Beta 4 by the ATP/ADP Ratio: Possible Implication in the Regulation of Actin Dynamics. Proc. Natl. Acad. Sci. 1993, 90 (11), 5034-5038.

https://doi.org/10.1073/pnas.90.11.5034.

(26) Yu, F. X.; Lin, S. C.; Morrison-Bogorad, M.; Atkinson, M. A.; Yin, H. L. Thymosin Beta 10 and Thymosin Beta 4 Are Both Actin Monomer Sequestering Proteins. J. Biol. Chem. 1993, 268 (1), 502-509.

(27) Didry, D.; Cantrelle, F.-X.; Husson, C.; Roblin, P.; Moorthy, A. M. E.; Perez, J.; Le Clainche, C.; Hertzog, M.; Guittet, E.; Carlier, M.-F.; et al. How a Single Residue in Individual $\beta$-Thymosin/WH2 Domains Controls Their Functions in Actin Assembly. EMBO J. 2012, 31 (4), 1000-1013. https://doi.org/10.1038/emboj.2011.461.

(28) Xue, B.; Leyrat, C.; Grimes, J. M.; Robinson, R. C. Structural Basis of Thymosin- 4/Profilin Exchange Leading to Actin Filament Polymerization. Proc. Natl. Acad. Sci. 2014, 111 (43), E4596E4605. https://doi.org/10.1073/pnas.1412271111.

(29) Carlier, M.-F.; Herzog, M.; Didry, D.; Renault, L.; Cantrelle, F.-X.; Van Heijenoort, C.; Knossow, M.; Guittet, E. Structure, Function, and Evolution of the Beta-Thymosin/WH2 (WASP-Homology2) Actin-Binding Module. Ann. N. Y. Acad. Sci. 2007, 1112 (1), 67-75. https://doi.org/10.1196/annals.1415.037.

(30) Renault, L.; Deville, C.; van Heijenoort, C. Structural Features and Interfacial Properties of WH2, $\beta$ Thymosin Domains and Other Intrinsically Disordered Domains in the Regulation of Actin Cytoskeleton Dynamics. Cytoskeleton 2013, 70 (11), 686-705. https://doi.org/10.1002/cm.21140. 


\section{TABLE}

Table 1. Binding affinities of natural actin ligands determined using a fluorescence polarization competition assay. $K_{d} S$ are indicated in nanomolar. Average values and standard deviations are shown.

\begin{tabular}{cccc}
\hline & G-buffer, $\mathrm{Mg}^{2+}$, ATP & G-buffer, $\mathrm{Mg}^{2+}$, ADP & G-buffer, $\mathrm{Ca}^{2+}$, ATP \\
\hline T $\beta 4$ & $127 \pm 11$ & $>8000$ & $520 \pm 80$ \\
swinholide A & $130 \pm 40$ & $79 \pm 22$ & $47 \pm 20$ \\
mycalolide B & $9.3 \pm 0.5$ & $<10$ & $<10$ \\
halichondramide & $10.7 \pm 2.6$ & $<10$ & $<10$ \\
\hline
\end{tabular}




\section{FIGURES}

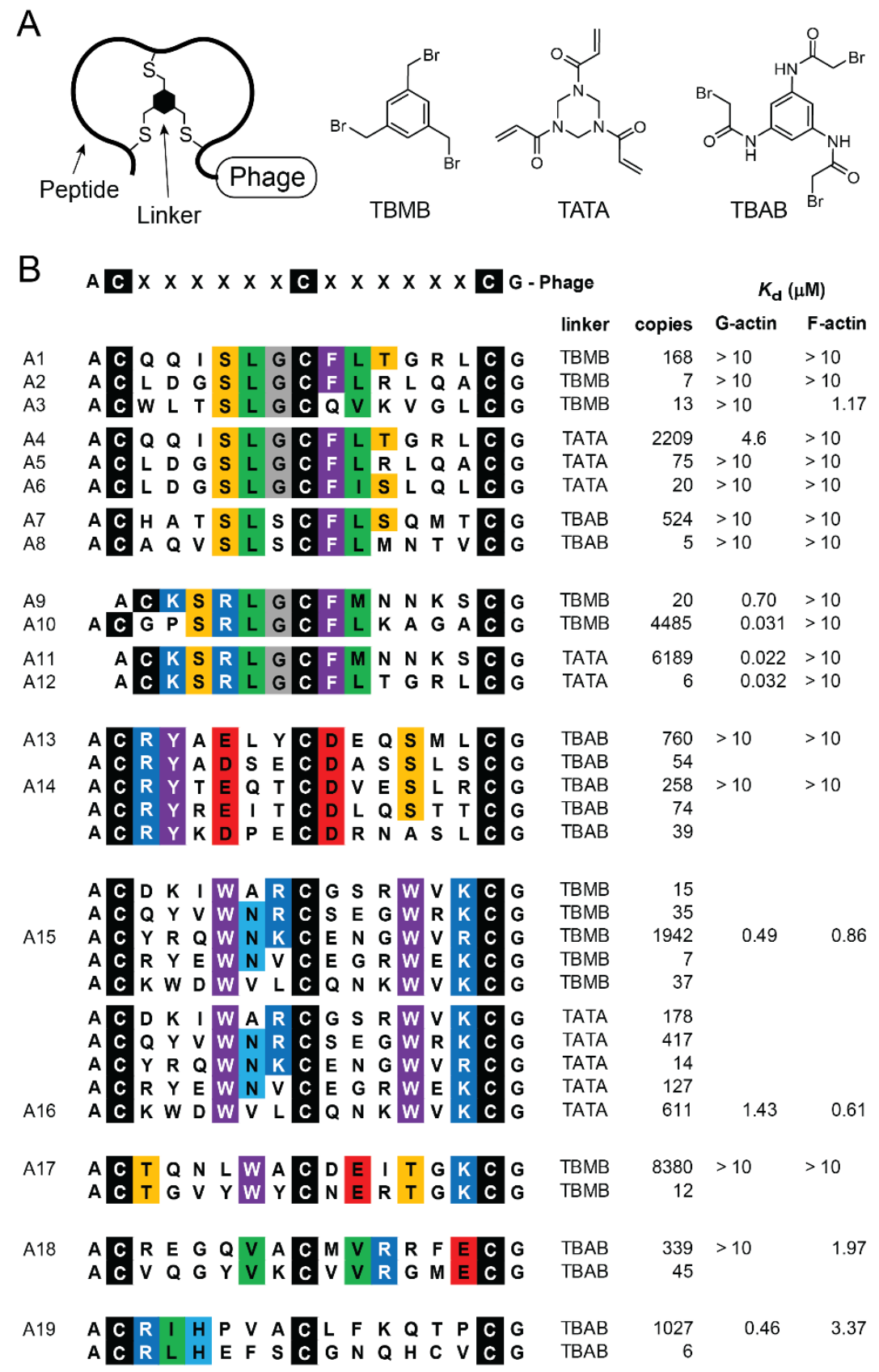

Figure 1. Phage selection of bicyclic peptides to G-actin. (A) Sequences and activity of isolated peptides. The peptides are aligned in groups based on sequence similarities, which are highlighted in color, and the chemical linker that was used in the selection. The abundance of peptides based on NGS data is shown. The binding affinity is indicated for the fluorescein-labeled bicyclic peptide to G- and F-actin, as measured by fluorescence polarization. 

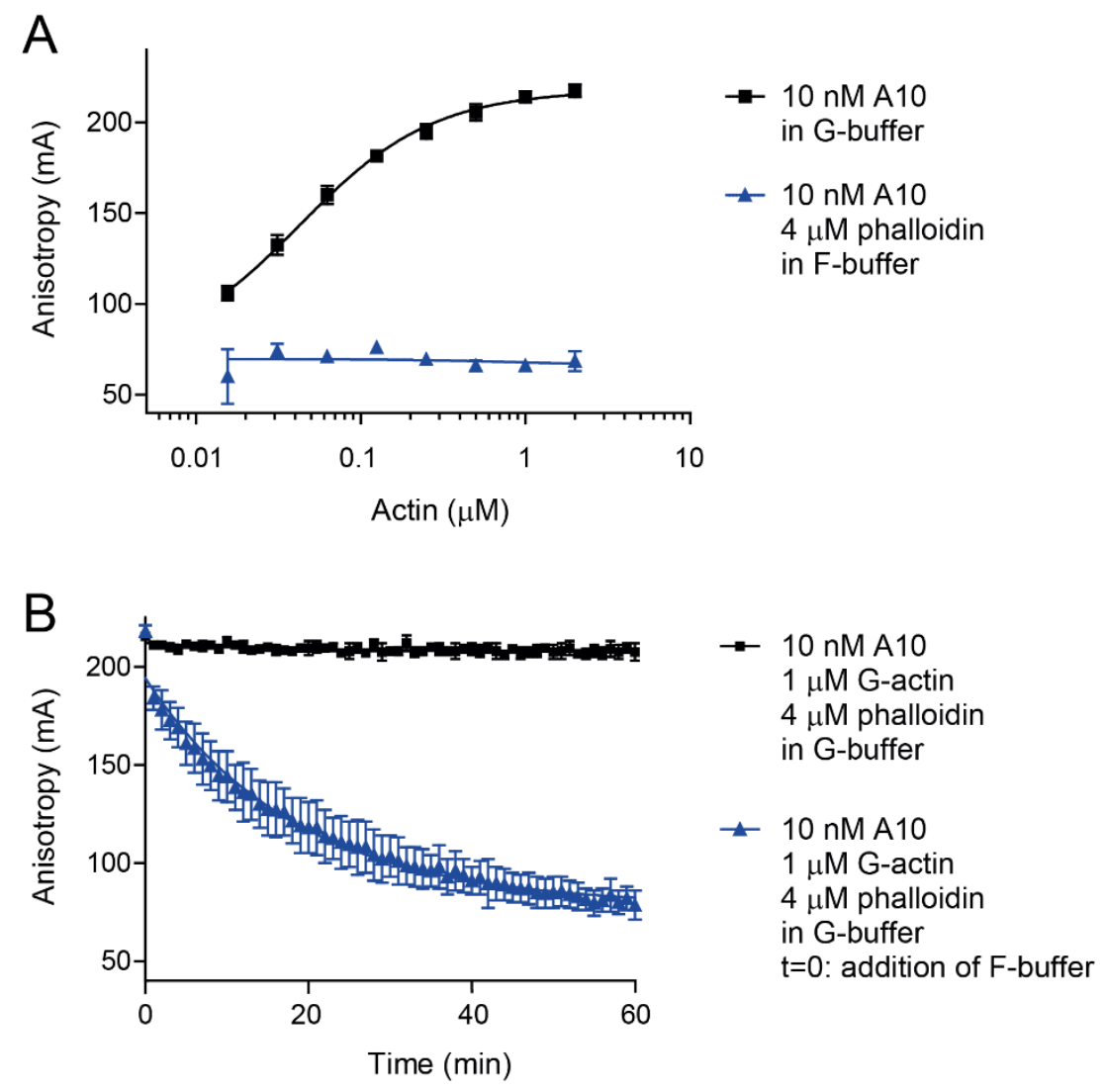

Figure 2. Binding of fluorescein-labeled bicyclic peptide $A 10$ to $G$ - and F-actin, as measured by fluorescence polarization. (A) Fluorescent peptide $\mathrm{A} 10$ at $10 \mathrm{nM}$ was incubated with increasing concentrations of monomeric actin (in G-buffer) or filamentous actin (in F-buffer supplemented with phalloidin). The addition of phalloidin to the F-buffer lowered the critical concentration necessary to ensure F-actin formation, especially at the lower actin concentrations. (B) Actin polymerization over time reduces the G-actin concentration, and consequently, the polarization of the fluorescent peptide A10. 
A
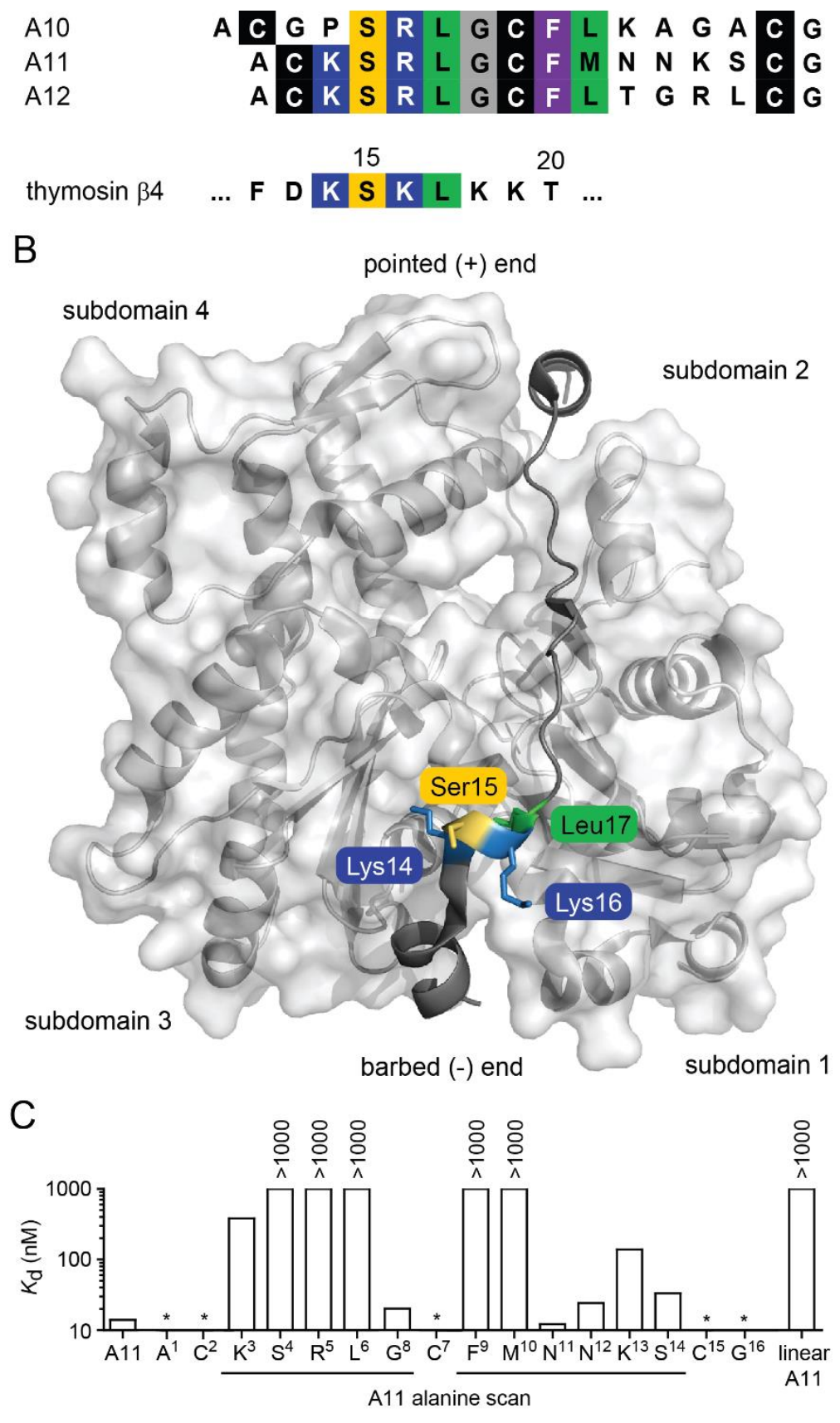

Figure 3. Sequence similarity between bicyclic peptides and T $\beta 4$. (A) Aligned sequences. (B) X-ray structure of T $\beta 4$ bound to Pichia actin (PDB entry 4PL7). Highlighted in color is the tetra-peptide motif that shares a sequence similarity with the bicyclic peptides. (C) Alanine scan of A11. ( $\left.{ }^{*}\right)$ For these amino acid positions, alanine mutants were not generated. 

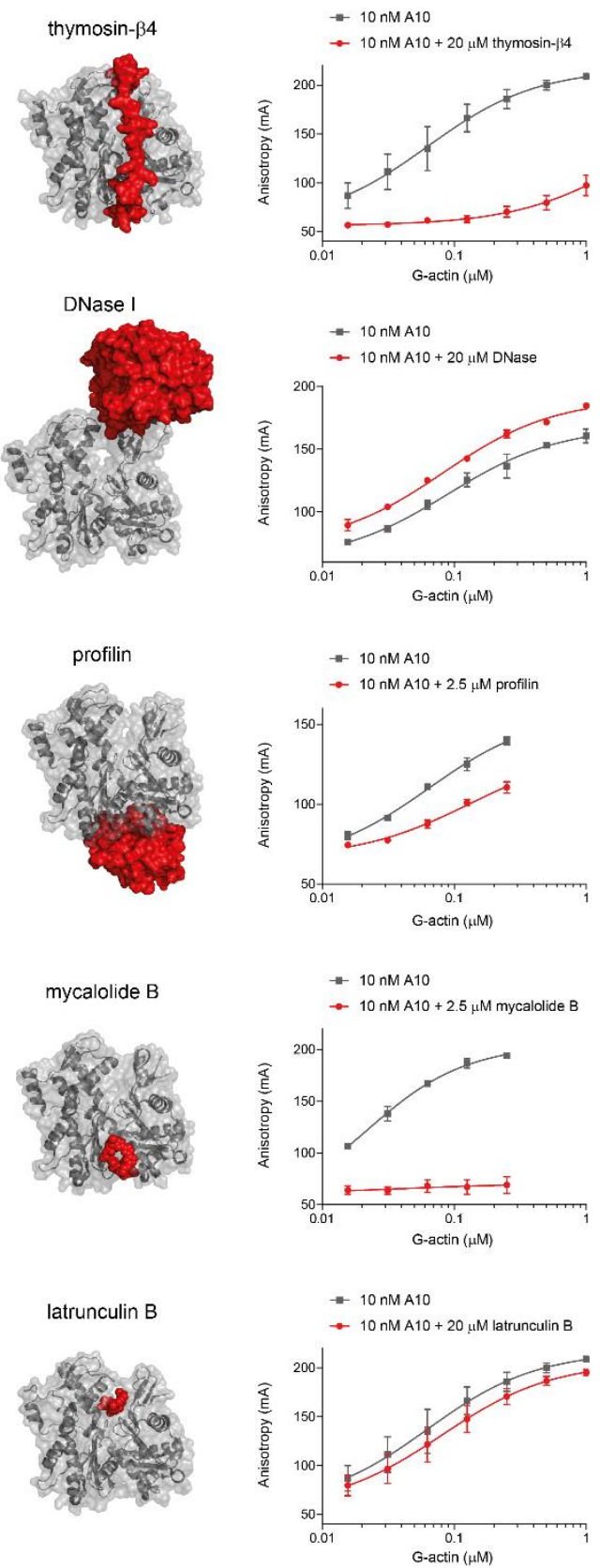

Figure 4. Competition between $\mathrm{A} 10$ and five natural actin ligands for binding to G-actin. Structures of the ligands (red) bound to actin (grey) are shown on the left. The fluorescence polarization of A10 in G-buffer at increasing actin concentrations. Actin binding was measured in the absence and presence of constant concentrations of natural actin ligands. For profilin and mycalolide B, we used a lower concentration (2.5 $\mu \mathrm{M})$ due to the limited amounts we had of these molecules. 


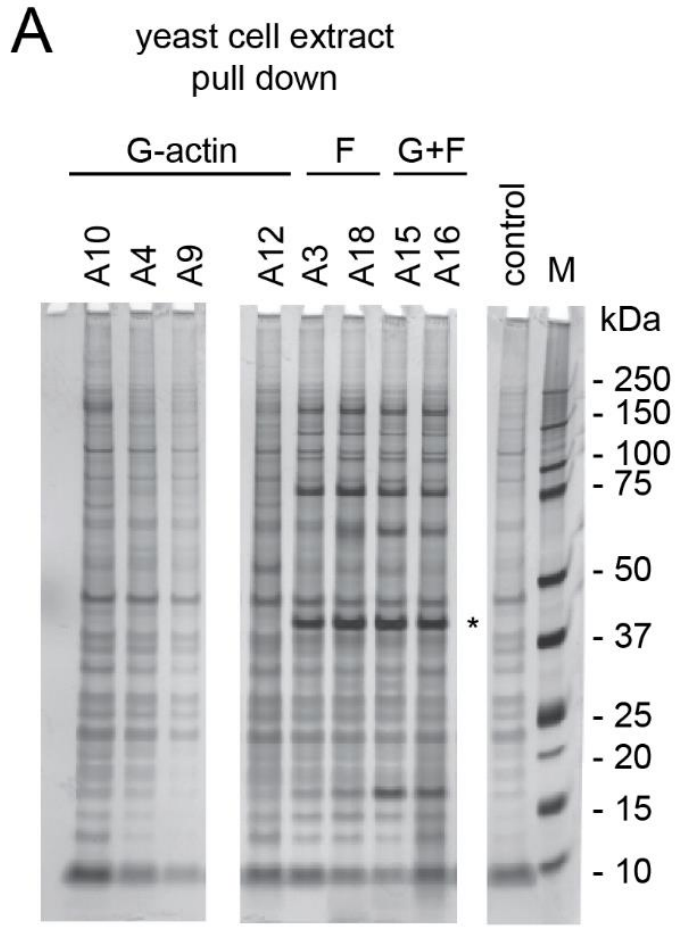

silver staining

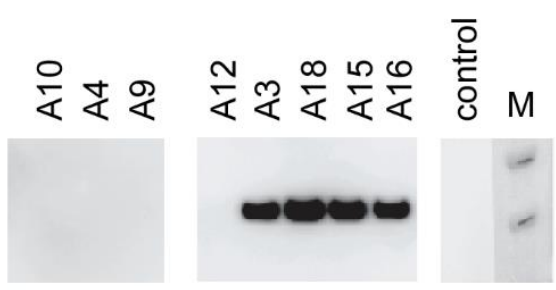

anti-actin
B
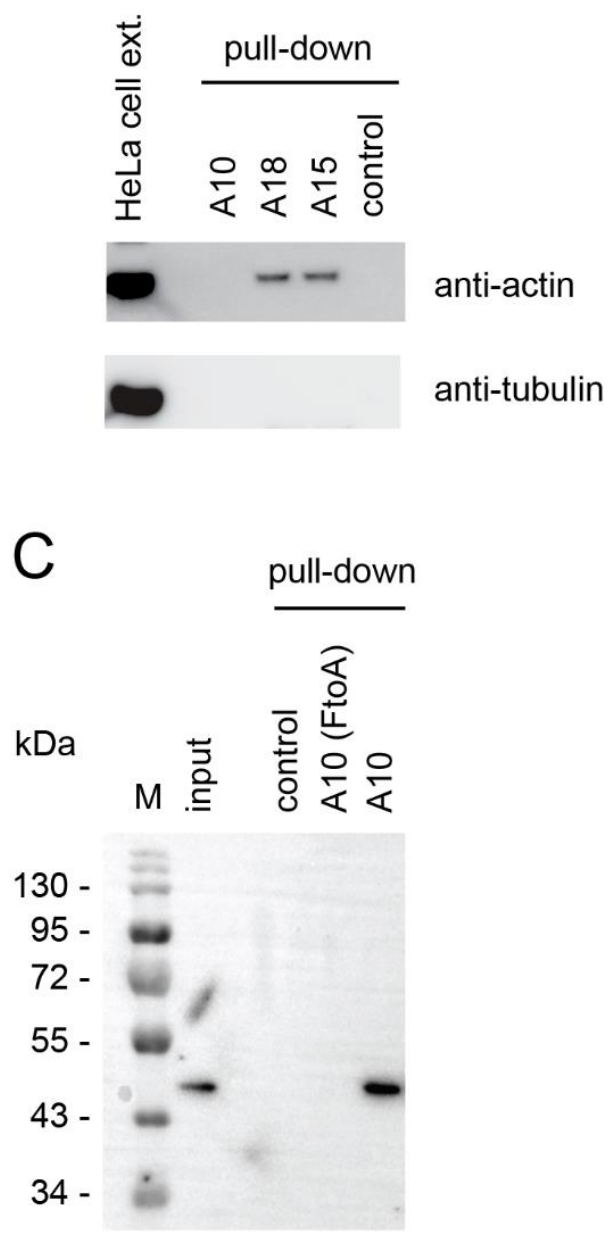

anti-actin

Figure 5. Application of bicyclic peptides as affinity ligands in pull-down experiments. Pull-down of actin from $(A)$ yeast and $(B)$ mammalian-cell extracts. Fluorescein-labeled bicyclic peptides specific for $\mathrm{G}$-actin (A4, A9, A10, A12), F-actin (A3, A18), or G- and F-actin (A15, A16) were immobilized on magnetic beads, incubated with cell lysate, washed, and eluted at acidic $\mathrm{pH}$. Proteins were separated by SDS-PAGE and analyzed by silver staining and Western blotting using antibodies for actin. Actin bands in (A) are indicated with as asterisk. Lanes indicated as "control" are protein captured with no peptide immobilized. (C) Pulldown of purified G-actin. Controls without G-actin (control) or peptide A10 with a point mutation, not binding G-actin (Phe10Ala), were included. 


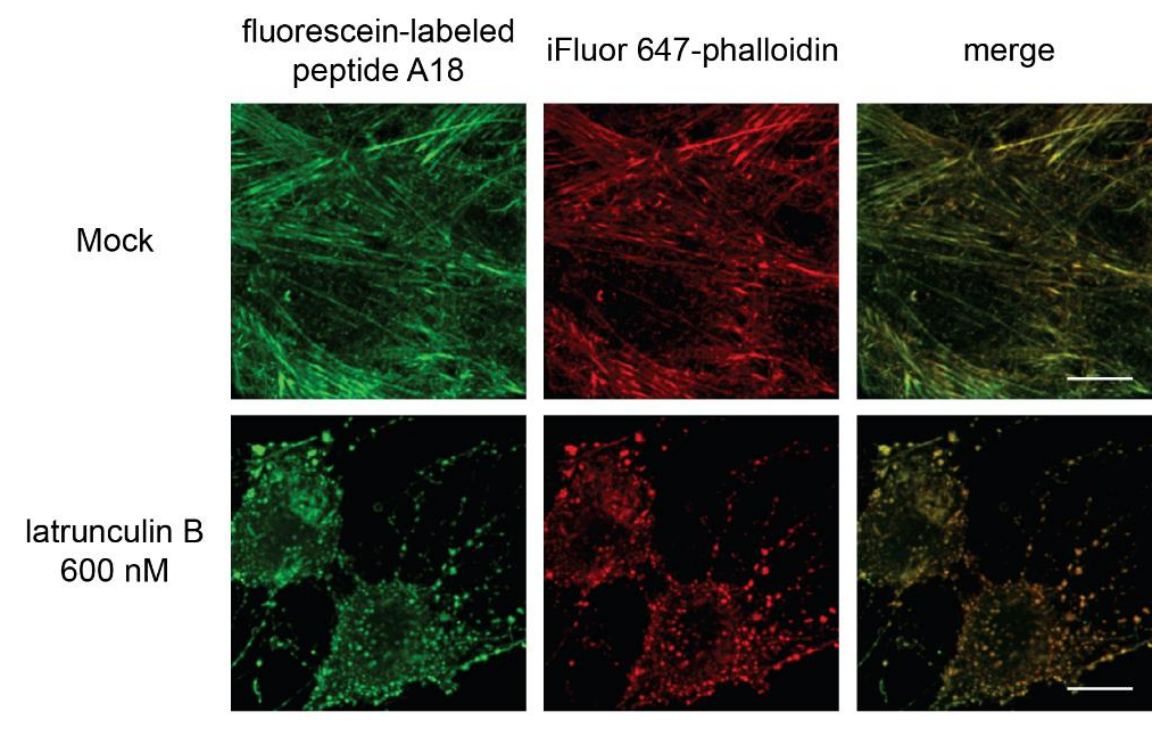

Figure 6. F-actin imaging in mammalian cells. HeLa cells treated with DMSO $(<0.025 \%)$ or the actin depolymerizing drug latrunculin B (600 nM) were stained with phalloidin (far red) and bicyclic peptide A18 (green). Shown are maximum intensity projections of a stack controlled for channel bleed through from green to far red. 

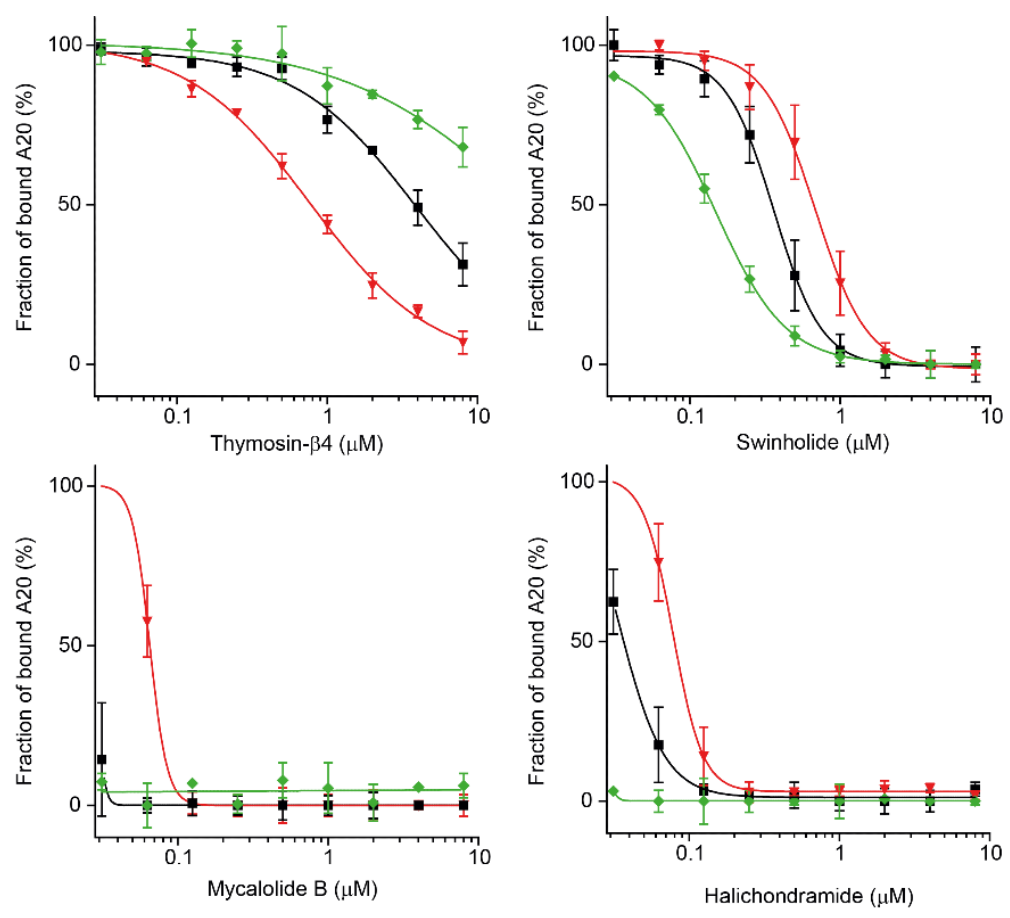

$\rightarrow$ G-buffer, Mg2+, ATP $\rightarrow$ G-buffer, $\mathrm{Mg}^{2+}$, ADP $\rightarrow$ G-buffer, $\mathrm{Ca}^{2+}$, ATP

Figure 7. Binding of natural actin ligands to monomeric actin measured in a competition experiment using the fluorescein-labeled peptide A20 as a probe. A20 (10 nM) was incubated with G-actin concentrations to reach 50 to $70 \%$ of the maximal change in fluorescence polarization (16 nM for the $\mathrm{Ca}^{2+} / \mathrm{ATP}$ condition, 35 $\mathrm{nM}$ for the $\mathrm{Mg}^{2+} / \mathrm{ATP}$ condition, $25 \mathrm{nM}$ for the $\mathrm{Mg}^{2+} / \mathrm{ADP}$ condition). The peptide was displaced by titrating in increasing concentrations of the actin ligands $T \beta 4$, swinholide, mycalolide $B$, and halichondramide. 
ToC Graphic

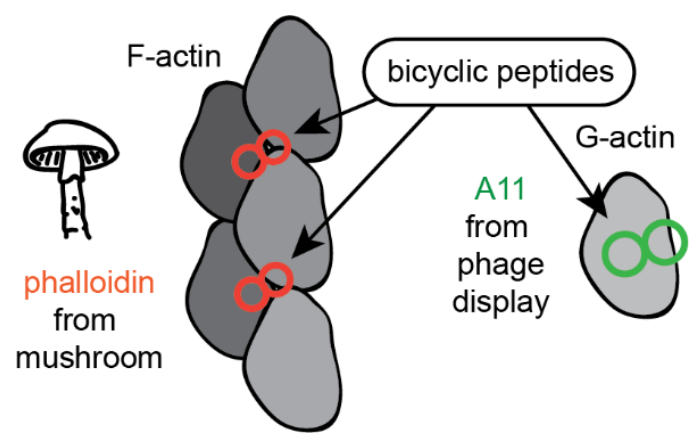

